\title{
Estudio comparativo de los hallazgos electrocardiográficos en colonias india y china en la ciudad de Iquique, Chile
}

\author{
Ma. Virginia Araya $A^{1,2}$, Pablo Vargas $M^{2,3}$, Rodrigo Araya $R^{2,3}$, Hargoon Samtani ${ }^{1,2}$, \\ Sebastián Córdova $\mathrm{O}^{2,3}$. \\ ${ }^{1}$ Centro Médico lquique \\ ${ }^{2}$ Red de Prevención Cardiovascular del Norte Grande, Iquique, Chile. \\ 3Interno Medicina, Universidad del Mar, Sede lquique.
}

\section{Resumen}

Introducción: La migración intrínsicamente implica la necesidad de adaptación a nuevos estilos de vida, siendo más radical cuando se trata de países tan lejanos y distintos, como India o China. Las variantes electrocardiográficas en estas culturas están siendo ampliamente estudiadas para entender cuando estos cambios son una anomalía o una adaptación fisiológica normal. Por este motivo se hace imperativo investigar sobre hallazgos electrocardiográficos de una población en constante aumento en nuestra sociedad.

Objetivo: Evaluar los hallazgos electrocardiográficos en 2 poblaciones de inmigrantes asiáticos con más de 2 años de residencia en Iquique, Chile. Metodología: Estudio analítico, descriptivo de corte transversal. Análisis de electrocardiograma (ECG) de 12 derivaciones, frecuencia cardíaca, intervalos PR, QTc y conclusiones diagnósticas del trazado.

Resultados: El grupo Chino (CHI) de 43 pacientes (37,2\% mujeres), edad de 39,5+13 años. Grupo Indio (IND) de 41 pacientes (48,8\% mujeres) con edad promedio de 38,6+10 años. CHI se encontró un $79,1 \%$ de trazados alterados con 55,8\% Bloqueo incompleto de Rama Derecha (BIRD), 11,6\% que mostraba signos de Bloqueo completo de Rama Derecha (BCRD), y un $11,6 \%$ presento un QTC alargado. IND el 75,6\% de los pacientes tenía un ECG dentro de los límites normales, los ECG alterados mostraban a un 14,6\% con BIRD y la pesquisa de QTC alargado fue de un $2 \%$ de la población.

Conclusión: Existe un alto porcentaje de pacientes chinos con electrocardiogramas alterados y especialmente con bloqueos de rama derecha, lo que hace necesario estudiar las causas y el rol que tendría la occidentalización y las anomalías genéticas en esto.

Palabras clave: Electrocardiografía, Migración, China, India.

\section{Characteristics of the electrocardiogram in subjects from Indian or Chinese descent living in Iquique, Chile.}

Background: Immigration implies the adaptation to new life styles and these changes are more complicated when countries are far way as is the case between Chile and both India an China. It is uncertain whether the electrocardiographic differences between these populations reflect adaptation changes or are just differences in normal patterns for each population.

Aim: to analyze characteristics of the electrocardiogram in subjects of Indian or Chinese descent living more than 2 years in Iquique, Chile.

Correspondencia: María Virginia Araya A.

Avenida Bilbao 3604. Iquique, Chile

Correo Electrónico: mvaraya@vtr.net 
Ma. V.Araya , P. Vargas, R. Araya , H. Samtani , S. Córdova.

Method: We analyzed the heart rate (HR), PR interval, and QTC in 12 lead EKGs from subjects of Indian or Chinese descent.

Results: 43 subjects (37.2\% women), aged $39.5 \pm 13$ years were included in the Chinese group (CHI); 41 subjects (48.8\% women), aged $38.6 \pm 10$ years were included in the Indian group (IND). $79.1 \%$ of EKGs in the CHI group were abnormal: incomplete right bundle branch block (RBBB) was present in $55.8 \%, 11.6 \%$ had complete RBBB and $11.6 \%$ a prolonged QT. On the other hand $75.6 \%$ of subjects in the IND group had a normal EKG; abnormalities included incomplete RBBB in $14.6 \%$ and prolonged QT in $2 \%$.

Conclusion: A high percentage of subjects of Chinese descent living for more than 2 years in lquique, Chile, exhibit electrocardiographic abnormalities, mainly RBBB. Further study is needed to elucidate whether genetic differences or adaptation to new conditions underlie these characteristics.

Key Words: immigration, electrocardiogram

Recibido el 9 de enero de 2009. Aceptado el 4 de mayo de 2009

\section{Introducción}

La migración intrínsicamente implica la necesidad de adaptación a una nueva cultura y por ende a un nuevo estilo de vida, siendo esto aún más radical cuando se trata de países tan lejanos y distintos a nuestra cultura occidental, como India o China1.2.

De acuerdo a los datos entregados por el Censo del 2002, destaca la gran presencia de la población inmigrante india y china en la ciudad de Iquique, donde habita un $43,1 \%$ y $15,5 \%$ de los inmigrantes indios y chinos de nuestro país, respectivamente 3 .

Actualmente existe una mortalidad por enfermedad cardiovascular que asciende al $36 \%$ en la República Popular China, principalmente por accidentes cerebrovasculares y derivados del ascenso de la hipertensión arterial en los últimos 30 años y el aumento de la sobrevida poblacional de 35 a 70 años 4 . Paralelamente, en la población residente en el sur de India las enfermedades cardiovasculares provocan el $24 \%$ de todas las muertes, principalmente por cardiopatía isquémica, que tienden a ser más altas en zonas urbanas y en clases sociales media y alta 5,6 .

En la literatura se describe una mayor prevalencia de factores de riesgo cardiovascular, y por lo tanto, de enfermedad cardiovascular en la población inmigrante, sobretodo en la asiática 7,8 , lo que según Misra se podría deber en el caso de la población india, a los altos niveles de lipoproteína "a" que

presentan 9 , siendo incrementada esta predisposición genética de ateratogénesis acelerada por los cambios en el estilo de vida señalados, dados por la propia occidentalización 1 .

Por otro lado, son varios los estudios que demuestran algunas variantes electrocardiográficas normales para determinadas etnias ${ }^{10}$, como por ejemplo, una mayor duración de la onda P o del QTc en la población china11, importantes a considerar al momento de evaluar a estos pacientes. Además, existe un estudio de Srikantia y cols. que demostró que no había diferencias significativas en la depresión del segmento ST en 941 sujetos estudiados; militares y civiles procedentes del sur de la India. Sin embargo, los cambios en la onda T en las derivadas DI, DIl y unipolares izquierdas presentaban cambios significativos en el grupo sobre los 50 años de edad6.

Pese a esto, desconocemos estudios que analicen la influencia que tiene la raza en estas variantes electrocardiográficas y el efecto sinérgico que tendría la occidentalización en este tema.

\section{Objetivo}

Evaluar los hallazgos electrocardiográficos en dos poblaciones de inmigrantes asiáticos con más de dos años de residencia en la ciudad de Iquique, Chile. 


\section{Metodología}

Estudio analítico, descriptivo de corte transversal, el cual analiza dos poblaciones inmigrantes con más de dos años de residencia en Chile, sin antecedentes personales de enfermedad cardiovascular ni consumo de medicamentos conocidos que pudieran alterar el electrocardiograma y que accedieron a participar voluntariamente de éste estudio: 41 indios provenientes del sur de India y 43 chinos provenientes de la República Popular China.

En ambos grupos se realizó un electrocardiograma de reposo de 12 derivaciones considerando las tres derivaciones de extremidades estándar y aumentadas (DI, DII y DIII, aVR, aVL, aVF), y las derivaciones precordiales ( $\mathrm{V} 1$ a $\mathrm{V} 6$ ), estandarizado con un trazado a $10 \mathrm{~mm} / \mathrm{mV}$ y $25 \mathrm{~mm} / \mathrm{seg}$, considerando los siguientes parámetros electrocardiográficos: Ritmo, Frecuencia Cardiaca, Eje Eléctrico, duración de intervalo PR, QRS, QT y QTc considerando este último como valores de referencia para hombre de hasta 450 mseg y mujeres hasta 470 mseg.

El estudio estadístico de los datos se realizó mediante un análisis descriptivo con cálculo de medias y frecuencias, prueba de $\mathrm{CHI}$-Cuadrado y t de Student.

\section{Resultados}

Se analizaron los datos de las dos poblaciones de inmigrantes: el grupo Chino (Grupo $\mathrm{CHI}$ ) estuvo compuesto por 16 mujeres $(37,2 \%)$ y 27 hombres $(62,8 \%)$ con una edad promedio de $39,5+13$ años. El grupo Indio (Grupo IND) lo constituyeron 20 mujeres $(48,8 \%)$ y 21 hombres $(51,2 \%)$, con una edad promedio de 38,6+10 años.

El grupo $\mathrm{CHI}$ mostró parámetros electrocardiográficos que tenían en promedio una frecuencia cardíaca de 71
Ipm, ritmo sinusal en el $100 \%$ de los pacientes donde un $11,6 \%$ presentó una Bradicardia Sinusal, el eje eléctrico de QRS se encontraba dentro de los límites normales en un $81,3 \%$ de los pacientes, un $4,65 \%$ tenía un eje con desviación a Izquierda y en el $11,6 \%$ eje eléctrico indeterminado. El $20,9 \%$ tenía un trazado electrocardiográfico dentro de los límites normales. Dentro del $79,1 \%$ de trazados alterados encontramos que el $11,6 \%$ mostraba signos de Bloqueo completo de Rama Derecha, el 55,8\% Bloqueo incompleto de Rama Derecha, un 2,3\% con Hipertrofia de Ventrículo Derecho, un 2,3\% para Hipertrofia ventricular izquierda, $11,6 \%$ presentó un QTc alargado, siendo el $18 \%$ de las mujeres y el $7,4 \%$ de los hombres, $2,3 \%$ con signos de Infarto de pared Anterior y un $4,7 \%$ de pacientes con signos de Isquemia.

El grupo IND tenía promedios electrocardiográficos que mostraron lo siguiente, una frecuencia Cardíaca $73 \mathrm{lpm}$, ritmo sinusal en el $100 \%$ de los pacientes donde 1 paciente $(2,4 \%)$ presentó una Bradicardia Sinusal, el eje eléctrico de QRS se mostró dentro de los límites normales en el $100 \%$ de los pacientes. El $75,6 \%$ de los pacientes del grupo IND tenía un electrocardiograma dentro de los límites normales, y mostrando alteración en el mismo en un $24,4 \%$ de los casos, dentro de los cuales un $14,6 \%$ presentó un Bloqueo incompleto de rama derecha, la pesquisa de QTc alargado fue de un $2 \%$ de la población total India correspondiendo a un sujeto de sexo masculino, un $7,3 \%$ tenía signos de isquemia y un $2,4 \%$ portaba una reacción auricular izquierda.

En el grupo $\mathrm{CHI}$ encontramos bloqueo de rama derecha en un $67,4 \%$ y en el grupo IND en un $14,6 \%$, siendo estadísticamente significativa esta diferencia $(p<0,000)$.

El promedio para el QT en colonia China fue de 0,38 mseg y en la India de 0,37 mseg, también significativo estadísticamente $(p<0,02)$. Tabla 1 y 2 .

Tabla 1: Resultados del análisis electrocardiográfico.

\begin{tabular}{lcc}
\hline VARIABLE & $\begin{array}{c}\text { Grupo IND } \\
(\mathrm{n}: 41)\end{array}$ & $\begin{array}{c}\text { Grupo CHI } \\
(\mathrm{n}: 43)\end{array}$ \\
\hline Ritmo Sinusal & 41 & 43 \\
Frecuencia Cardiaca (Ipm) & $73+9,9$ & $71+10 ., 1$ \\
Bradicardia Sinusal & 1 & 5 \\
Eje QRS Normal & 41 & 35 \\
\hline
\end{tabular}


Ma. V.Araya, P. Vargas, R. Araya , H. Samtani , S. Córdova.

Tabla 2. Hallazgos de Electrocardiogramas alterados.

\begin{tabular}{lcc}
\hline VARIABLE & $\begin{array}{c}\text { Grupo IND } \\
(\mathbf{n}: \mathbf{4 1})\end{array}$ & $\begin{array}{c}\text { Grupo CHI } \\
(\mathbf{n}: \mathbf{4 3})\end{array}$ \\
\hline EKG normal (pacientes) & 31 & 9 \\
EKG Alterado & 10 & 34 \\
BIRD & 6 & 24 \\
BCRD & 0 & 5 \\
Hipertrofia Ventricular & 0 & 2 \\
Reaccion Auricular & 1 & 0 \\
Infarto/lsquemia & 3 & 3 \\
Prolongacion QTC & 1 & 5 \\
\hline
\end{tabular}

BIRD: Bloqueo incompleto de rama derecha.

BCRD: Bloqueo completo de rama derecha.

\section{Discusión}

Existe en nuestro país un aumento de la población de origen asiático, que generalmente se establecen con fines comerciales en nuestra región. Por lo cual, establecer su estado de salud es importante, toda vez que se trate de sujetos jóvenes que luego presentan patologías que se deben establecer de acuerdo a un patrón determinado a su origen racial.

El electrocardiograma, siendo un examen antiguo y presente hasta hoy como una gran herramienta diagnóstica, ha sido nuestro patrón de estudio en estas dos poblaciones de 41 indios y 43 chinos. A pesar del número de pacientes estudiados, estos fueron seleccionados de acuerdo a edad, sexo y raza, midiendo en los electrocardiogramas la frecuencia cardíaca, que fue similar en ambos grupos, con un $100 \%$ de los individuos en ritmo sinusal.

Lo llamativo de este estudio fue el hallazgo de bloqueos de rama derecha en la población china, con un significancia altamente estadística, identificando $55,8 \%$ de bloqueos incompletos de rama derecha y $11,6 \%$ de bloqueos completos de rama derecha en la población china, mientras que en la población india un $14,6 \%$ de bloqueos incompletos de rama derecha y $0 \%$ de bloqueos completos de rama derecha. Cabe señalar que en la bibliografía revisada no se describe una mayor incidencia de bloqueos de rama derecha en la población china no inmigrante, haciendo alusión sólo a una mayor duración de la onda P y QTc 11.

Uno de los aspectos importantes del hallazgo de bloqueo de rama derecha en inmigrantes chinos es por su relación con el Síndrome de Brugada, ya que se ha sugerido que este síndrome es más frecuente en los pacientes de origen asiático12,13. Como los cambios electrocardiográficos característicos de este síndrome suelen ser intermitentes, el bloqueo de rama derecha puede ser la única alteración en el electrocardiograma que permita sospechar este cuadro, especialmente si el paciente tiene síntomas o historia familiar sugerente. Igualmente, el uso de algunos fármacos que pueden desenmascarar un síndrome de Brugada y potencialmente gatillar arritmias malignas y muerte súbita, como las drogas clase Ic flecainide y propafenona, debieran ser evitados al menos que no existan elementos para sospechar ese síndrome.

\section{Conclusiones}

Llama la atención el alto porcentaje de pacientes chinos con electrocardiogramas alterados y especialmente con bloqueos de rama derecha.

Se hace necesario el diseño de estudios que analicen las causas de esta mayor cantidad de bloqueos de rama en la población china y el rol que jugaría la occidentalización y las anomalías genéticas en ésto, además de la identificación de otros factores condicionantes.

Pese a no contar con casos de Síndrome de Brugada en nuestra serie, la alta cantidad de bloqueos de rama identificados obliga a optimizar la pesquisa de esta patología, y tener especial precaución en esta población, respecto a la administración de antiarrítmicos de clase IC. 
La poca evidencia publicada corrobora la necesidad de evaluar las poblaciones inmigrantes de forma holística, no dejando de lado los cambios per se que trae consigo la occidentalización, ya que pueden ser fundamentales a la hora de tomar decisiones en el manejo de estos pacientes.

\section{Referencias}

1. ARAYA MV, SAMTANI H, FERRADA MS, VARGAS P, ARAYA R, ARRIETA MA, et al. Impacto de la occidentalización sobre los factores de riesgo cardiovascular en inmigrantes asiáticos residentes en el norte grande de Chile. Rev Chil Cardiol 2009: 28; 45-50.

2. BRODOV Y, MANDELZWEIG L, BOYKO V, BEHAR S. Is immigration associated with an increase in risk factors and mortality among coronary artery disease patients? A cohort study of 13,742 patients. Isr Med Assoc J 2002; 4: 326-30.

3. MARTINEZ PIZARRO J. Breve examen de la inmigración en Chile según los datos generales del Censo 2002. Celade- CEPAL.

4. BRAUNWALD, ZIPES, LIBBY, BONOW. Braunwald's Heart Disease: A Texbook of Cardiovascular Medicine $7^{a}$ Edition2006 Vol 1 The changes patterns of cardiovascular disease in China. Chapter 1: 10-11.

5. RAMACHANDRAN A, SNEHALATHA C, SATYAVANI KM SIVASANKARI S, VIJAY V. Metabolic syndrome in urban Asian Indian adults--a population study using modified ATP III criteria. Diabetes Res Clin Pract 2003; 60: 199-204.

6. SRIKANTIA SG, PADMAVATI S, GOPALAN C. The Electrocardiogram in Some Indian Population Groups. Circulation. 1964; 29: 118-23.

7. ANAND SS, YUSUF S, VUKSAN V, DEVANESEN S, TEO KK,
MONTAGUE PA, et al. Differences in risk factors, atherosclerosis, and cardiovascular disease between ethnic groups in Canada: the Study of Health Assessment and Risk in Ethnic groups (SHARE). Lancet. 2000; 356: 279-84.

8. PATEL JV, VYAS A, CRUICKSHANK JK, PRABHAKARAN D, HUGHES E, REDDY KS, et al. Impact of migration on coronary heart disease risk factors: Comparison of Gujaratis in Britain and their contemporaries in villages of origin in India. Atherosclerosis. 2006: 185; 297-306.

9. MISRA A. Atherosclerosis in indians and lipoprotein (a). J Assoc Physicians India. 1999; 47: 313-7.

10. MACFARLANE PW, MCLAUGHLIN SC, DEVINE B, YANG TF. Effects of age, sex, and race on ECG interval measurements. J Electrocardiol. 1994; 27: 14-9.

11. WU J, KORS JA, RIJNBEEK PR, VAN HERPEN G, LU Z, $\mathrm{XU}$ C. Normal limits of the electrocardiogram in Chinese subjects. Int J Cardiol. 2003; 87: 37-51.

12. BENITO B, BRUGADA R, BRUGADA J, BRUGADA P. Brugada Syndrome. Prog Cardiovasc Dis 2008; 51: 1-22.

13. MOK NS, PRIORI SG, NAPOLITANO C, CHAN KK, BLOISE R, CHAN HW, et al. Clinical profile and genetic basis of Brugada Syndrome in the Chinese population. 2004; 10: 32-7. 